\section{Birthday of a syndrome: 50 years anniversary of Smith-Lemli-Opitz Syndrome}

European Journal of Human Genetics (2015) 23, 277-278; doi:10.1038/ejhg.2014.87; published online 14 May 2014

\section{HISTORY OF THE SMITH-LEMLI-OPITZ SYNDROME}

Fifty years ago, the Smith-Lemli-Opitz Syndrome (SLOS) was described in three male patients by pediatricians David W Smith, Luc Lemli and John Opitz at the University of Wisconsin, USA, for the first time. ${ }^{1}$ It was designed as a clinical description of all patients who had microcephaly and hypogenitalism.

SLOS was initially named RSH, a non-descriptive acronym of the first letters of the original patients' surnames. Its name was later changed in order to honor the three geneticists who first described this disorder. Soon after, it was usual practice to distinguish between a less-expressed form of SLOS, named type I, and the more severe type II form. Since the findings of the molecular cause of SLOS, it is clear that the subdivision into these two forms is inappropriate. The difference between type I and type II SLOS can be explained as a consequence of the underlying mutations.

Just 30 years later, Tint et al published their measurements of neutral sterols in the plasma of five patients with SLOS and found abnormally low concentrations of cholesterol, but greater than 1000-fold increases in the level of 7-dehydrocholesterol, the immediate precursor of cholesterol in the Kandutsch-Russell pathway for biosynthesis of cholesterol. This step in the biosynthesis of cholesterol is catalyzed by $\Delta 7$-dehydrocholesterol reductase. Subsequently, the underlying DHCR7 gene was identified and cloned in $1998 .^{3}$

\section{CLINICAL PHENOTYPE AND VARIABILITY}

Typical symptoms of SLOS include 2, 3 toe syndactyly and facial dysmorphisms as, for example, anteverted nares, which are key features of these patients. The characteristic craniofacial appearance of SLOS patients is independent from the clinical or biochemical severity. ${ }^{4}$ Even the least-affected patients may show a characteristic facial phenotype. Individuals with SLOS have a number of neurodevelopmental problems, which are a consistent part of the syndrome. The most commonly observed brain abnormalities affect midline structures, such as the corpus callosum, intraventricular septum, and interior cerebellar vermis, ${ }^{5}$ and, if present, may contribute to the neurodevelopmental symptoms.

The disease phenotype in SLOS is mainly thought to be caused by a lack of cholesterol and accumulation of 7- and 8-DHC (dehydrocholesterol) during embryogenesis. ${ }^{2}$ But as the biochemical pathogenesis is still incompletely understood, no proven therapy for this disease exists to date.

Roullet et $a l^{6}$ hypothesized that accumulation of cholesterol precursors might lead to a preference for other additional sterol pathways. They showed that SLOS patients exhibit an altered urinary excretion of 3-methylglutaconic acid (3MGC) and a diversion of the sterol precursor farnesyl-PP toward long-chain isoprenoids. No evidence for mevalonate shunting was demonstrated in moderately affected SLOS patients. On the other hand, significant pathophysiology regarding SLOS phenotype occurs in the brain of SLOS patients, which might be explained by the various distribution patterns of cholesterol, 7-DHC and DHCOE (oxysterol metabolite of 7-DHC) in the brain regions. ${ }^{7}$

By comparing the SLOS patients' clinically described severity scores $^{8}$ along with the corresponding biochemical data and their genotypes, a clear correlation between genotypes and phenotypes ${ }^{9}$ became evident. This correlation was confirmed in Polish patients. ${ }^{10}$ However, SLOS patients with the same genotype did show a wide range in phenotype and clinical severity. This led to the assumption that additional modifying factors, apart from the DHCR7 genotype, influence the SLOS phenotype and disease severity. A surprising finding in SLOS was the modifying effect of apoE on the clinical severity of the disease. It was shown that disease severity varies significantly depending on whether the apoE alleles $\varepsilon 2$, $\varepsilon 3$ or $\varepsilon 4$ were carried by the respective patient's mother ${ }^{11}$ (Figure 1). Another possible modifier of severity might be ABCA1. ${ }^{12}$

\section{SPECTRUM OF DHCR7 MUTATIONS IN SLOS PATIENTS}

As discussed, SLOS is a metabolic and malformation disorder caused by mutations in the DHCR7 gene (7-dehydrocholesterol reductase, reference sequences: NG_012655, NM_001360.2, LRG_340) on chromosome 11. The first pathogenic mutations in SLOS patients were detected by our group in Innsbruck, Austria, as well as by the groups of Wassif and of Waterham in $1998 .{ }^{13-15}$ So far, more than 130 disease-causing mutations have been described and all reported patients in the literature are included in a DHCR7 database (http:// databases.lovd.nl/shared/genes/DHCR7). The interpretation of newly detected variants, genotypes and phenotypic severity is more accurately possible using such well-defined databases.

Despite the fact that a large number of mutations have been identified, the majority of the SLOS cases are caused by the five most abundant mutations. The most common mutation is the splice site mutation c.964-1G >C (transcript reference sequence: NM_001360.2, frequency of $29 \%$ in SLOS alleles), ${ }^{9}$ which leads to an insertion of $134 \mathrm{bp}$ between exons 8 and 9. Other frequently observed mutations are c.1210C $>$ A (p.(Arg404Cys) with 11\%), c.976G $>$ T (p.(Val326Leu), 7\%), c.452G $>$ A (p. $\left.\left(\operatorname{Trp} 151^{\star}\right), 8 \%\right)$ and c.278C $>$ T (p.(Thr93Met), 8\%). ${ }^{9}$ Interestingly, specific mutation spectra are prevalent in different European populations. The occurrence of mutations with surprisingly high carrier frequencies in various populations was thought to be an indication for positive selection of those mutations. However, subsequent research has shown that the time span since the first occurrence of the most common mutations (c.964-1G $>$ C, p. $($ Trp151*) and p. (Thr93Met)) is sufficient (about 100 generations for c.964-1G $>C$ and p.(Trp151*), and 200 generations for $\mathrm{p}$.(Thr93Met)) to explain their frequencies $(1: 100$ for c.964-1G > C) by genetic drift alone ${ }^{16}$ (see also Figure 2).

\section{SLOS THERAPIES}

The basic therapeutic approach to SLOS is treatment by cholesterol substitution. It has been suggested that adding HMG-CoA reductase inhibitors like simvastatin might lessen the disease severity ${ }^{17}$ because simvastatin can cross the blood-brain barrier. The principal problem 

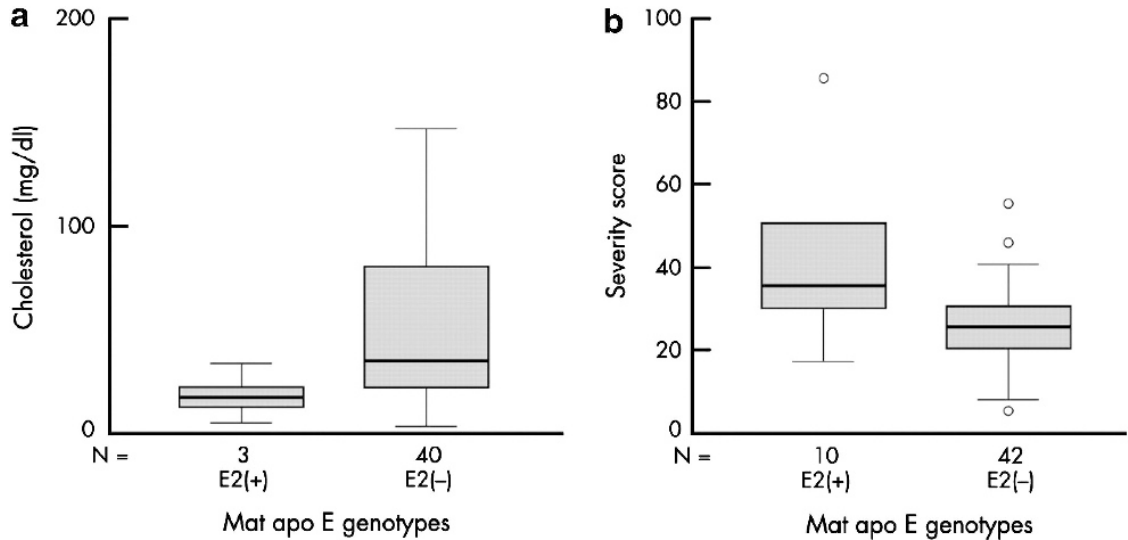

The E2(+) group includes individuals with genotype apo E2/E3 and with genotype E2/E4 The E2(-) group includes individuals with genotype apo E3/E4 and with genotype E4/E4

Figure 1 Box plot of correlation of maternal apo E genotype (E2 present versus absent) with cholesterol levels (a) and disease severity (b) in patients with SPOL (Figure 3 fromWitsch-Baumgartner et $a^{11}$ ).

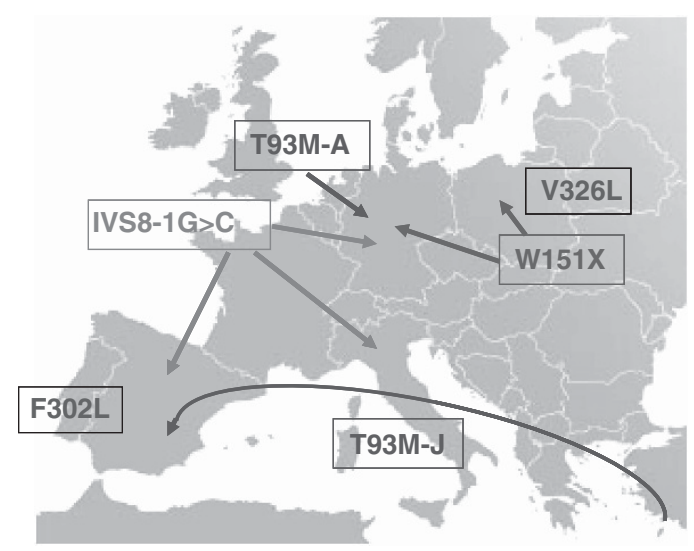

Figure 2 Interpretation of the origin and proliferation of SLOS mutations: Frequent mutations, such as c.964-1G > C, p.(Trp151*) and p.(Thr93Met), are likely to be founder mutations originally emerging in North-West Europe, Eastern Europe, and the Eastern Mediterranean region, respectively (p.(Thr93Met) with J haplotype background).

of the disease is that the clinical phenotype is due to lack of cholesterol during embryogenesis; hence therapy would be needed at that moment.

New therapeutic approaches aim to inhibit the formation of the toxic precursor 7-DHC or 7-DHC-derived oxysterols by antioxidant supplementation. Antioxidants, specifically vitamin E supplementation, can effectively inhibit the peroxidation of 7-DHC in SLOS fibroblasts and newborn Dhcr7-KO mice. ${ }^{18}$

Fifty years after the first description of SLOS the molecular base seems to be well established, whereas the clinical and biochemical pathophysiology behind the disease is less clear, as cholesterol serves various complicated functions in metabolism. In order to achieve constructive therapies for SLOS patients, it is necessary to continue research on this metabolic malformation syndrome.

\section{CONFLICT OF INTEREST}

The authors declare no conflict of interest.
Martina Witsch-Baumgartner* and Barbara Lanthaler Department of Medical Genetics, Molecular and Clinical Pharmacology, Division of Human Genetics, Innsbruck, Austria E-mail: witsch-baumgartner@i-med.ac.at

1 Smith DW, Lemli L, Opitz JM: A newly recognized syndrome of multiple congenital anomalies. J Pediatr 1964; 64: 210-217.

2 Tint GS, Irons M, Elias ER et al: Defective cholesterol biosynthesis associated with the Smith-Lemli-Opitz syndrome. N Engl J Med 1994; 330: 107-113.

3 Moebius FF, Fitzky BU, Lee JN, Paik YK, Glossmann H: Molecular cloning and expression of the human delta7-sterol reductase. Proc Natl Acad Sci USA 1998; 95: 1899-1902.

4 Nowaczyk MJ, Tan M, Hamid JS, Allanson JE: Smith-Lemli-Opitz syndrome: objective assessment of facial phenotype. Am J Med Genet A 2012; 158A: 1020-1028.

5 Lee RW, Conley SK, Gropman A, Porter FD, Baker EH: Brain magnetic resonance imaging findings in Smith-Lemli-Opitz syndrome. Am J Med Genet A 2013; 161: 2407-2419.

6 Roullet JB, Merkens LS, Pappu AS et al: No evidence for mevalonate shunting in moderately affected children with Smith-Lemli-Opitz syndrome. J Inherit Metab Dis 2012; 35: 859-869.

$7 \mathrm{Xu} \mathrm{L}$, Mirnics K, Bowman AB et al: DHCEO accumulation is a critical mediator of pathophysiology in a Smith-Lemli-Opitz syndrome model. Neurobiol Dis 2012; 45: 923-929.

8 Kratz LE, Kelley RI: Prenatal diagnosis of the RSH/Smith-Lemli-Opitz syndrome. Am J Med Genet 1999; 82: 376-381.

9 Witsch-Baumgartner M, Fitzky BU, Ogorelkova $\mathrm{M}$ et al: Mutational spectrum in the Delta7-sterol reductase gene and genotype-phenotype correlation in 84 patients with Smith-Lemli-Opitz syndrome. Am J Hum Genet 2000; 66: 402-412.

10 Ciara E, Nowaczyk MJ, Witsch-Baumgartner M et al: DHCR7 mutations and genotypephenotype correlation in 37 Polish patients with Smith-Lemli-Opitz syndrome. Clin Genet 2004; 66: 517-524.

11 Witsch-Baumgartner M, Gruber M, Kraft HG et al: Maternal apo E genotype is a modifier of the Smith-Lemli-Opitz syndrome. J Me Genet 2004; 41: 577-584.

12 Lanthaler B, Steichen-Gersdorf E, Kollerits B, Zschocke J, Witsch-Baumgartner M: Maternal ABCA1 genotype is associated with severity of Smith-Lemli-Opitz syndrome and with viability of patients homozygous for null mutations. Eur J Hum Genet 2013; 21: 286-293.

13 Fitzky BU, Witsch-Baumgartner M, Erdel $M$ et al: Mutations in the Delta7-sterol reductase gene in patients with the Smith-Lemli-Opitz syndrome. Proc Natl Acad Sci USA 1998; 95: 8181-8186.

14 Wassif CA, Maslen C, Kachilele-Linjewile $S$ et al: Mutations in the human sterol delta7-reductase gene at 11q12-13 cause Smith-Lemli-Opitz syndrome. Am J Hum Genet 1998; 63: 55-62.

15 Waterham HR, Wijburg FA, Hennekam RC et al: 'Smith-Lemli-Opitz syndrome is caused by mutations in the 7-dehydrocholesterol reductase gene,'. Am J Hum Genet 1998; 63: 329-338.

16 Witsch-Baumgartner M, Schwentner I, Gruber M et al: Age and origin of major SmithLemli-Opitz syndrome (SLOS) mutations in European populations. J Med Genet 2008; 45: 200-209.

17 Haas D, Garbade SF, Vohwinkel C et al: Effects of cholesterol and simvastatin treatment in patients with Smith-Lemli-Opitz syndrome (SLOS). J Inherit Metab Dis 2007; Vol. 30: 375-387.

18 Korade Z, Xu L, Harrison FE et al: Antioxidant supplementation ameliorates molecular deficits in Smith-Lemli-Opitz Syndrome. Biol Psychiatry 2014; 75: 215-222. 